\title{
Relative spectral theory and measure-theoretic entropy of gaussian extensions
}

\author{
by \\ J.-P. Thouvenot (Paris) \\ Dedicated to M. Misiurewicz on the occasion of his 60th birthday
}

\begin{abstract}
We describe the natural framework in which the relative spectral theory is developed. We give some results and indicate how they relate to two open problems in ergodic theory. We also compute the relative entropy of gaussian extensions of ergodic transformations.
\end{abstract}

1. Introduction. Let $(X, \mathcal{A}, m, T)$ denote a dynamical system; this means that $T$ is an invertible measure preserving transformation of the Lebesgue space $(X, \mathcal{A}, m)$. As usual, we shall frequently condense the previous notation to $(X, T)$ or even, when there is no ambiguity, to $T$. The unitary operator $U_{T}$ induced by $T$ on $L^{2}(X)$, defined by $f(x) \mapsto f(T(x))$, the Koopman operator, is a powerful tool to study questions related to the transformation $T$. It is obvious (and well known) that if two transformations $S$ and $T$ are isomorphic, then $U_{T}$ and $U_{S}$ are unitarily equivalent. It is therefore possible, just spectral considerations relating to Koopman operators, to show that some transformations cannot be isomorphic. Furthermore, in the case where the Koopman operators have purely atomic spectral measures, unitary equivalence of the Koopman operators implies isomorphism of the corresponding transformations. The spectral theorem gives a complete set of invariants for unitary equivalence. (For a treatment of spectral theory see, for instance [8].) If the spectral measures of the Koopman operators associated to two transformations $S$ and $T$ are mutually singular, then $S$ and $T$ are disjoint in the ergodic-theoretical sense (first defined by Furstenberg). The spectral multiplicity of the Koopman operator is by itself a powerful invariant. For instance, if a transformation is rank one (that is, for every

2010 Mathematics Subject Classification: 37A05, 37A35, 37A50, 37A30.

Key words and phrases: joining, entropy, Bernoulli factor, Rokhlin cocycle, Hilbert bundle, $K$-automorphism, discrete spectrum. 
finite partition $P$ and every $\epsilon$, there exists a Rokhlin tower such that every set in $P$ can be approximated by some union of levels of the tower to within $\epsilon$ for the distance which is the measure of symmetric difference), its cartesian square cannot be rank one. This is because the Koopman operator associated to a rank one transformation has spectral multiplicity one while the Koopman operator associated to a cartesian square has spectral multiplicity at least two.

The relative spectral theory deals with the following objects. We are given $(X, \mathcal{A}, m, T)$ as before, and a family of separable Hilbert spaces $H_{x}$ associated to every $x \in X$ equipped with a measurable structure coming from a countable fundamental family of sections (vector fields). The set of measurable sections is denoted $\int^{\oplus} H_{x} d m(x)$ and is naturally endowed with a structure of module over $L^{\infty}(X)$ (see [3, p. 138]). In most circumstances in this paper, $H_{x}$ will be a copy of a fixed separable Hilbert space $H$, and the fundamental family of sections generating the measurable structure will be the family of constant vectors $v_{i}(x), i \in \mathbb{N}$, where every $v_{i}(x)$ is a copy of $v_{i}$, the family $v_{i}, i \in \mathbb{N}$, forming an orthonormal basis of $H$. We will refer to this as the standard Hilbert bundle. Two Hilbert bundles $\int^{\oplus} H_{1, x} d m(x)$ and $\int^{\oplus} H_{2, x} d m(x)$ are said to be isomorphic if there is a measurable family $U_{x}$ of unitary isomorphisms $H_{1, x} \rightarrow H_{2, x}$ (that is, for every $v(x) \in \int^{\oplus} H_{1, x} d m(x)$, $\left.U_{x}(v(x)) \in \int^{\oplus} H_{2, x} d m(x)\right)$. In case $\int^{\oplus} H_{1, x} d m(x)$ is the constant field of Hilbert spaces as above, if we equip $\mathcal{U}(H)$, the set of unitary operators on $H$, with the Borel structure associated to the topology of strong convergence, a measurable map from $(X, \mathcal{A})$ to $\mathcal{U}(H), x \mapsto U_{x}$, will naturally provide the family $H_{2, x}=U_{x}\left(H_{1, x}\right), x \in X$, with a measurable structure such that $U_{x}$ defines an isomorphism between $\int^{\oplus} H_{1, x} d m(x)$ and $\int^{\oplus} H_{2, x} d m(x)$.

A unitary cocycle $U$ above $(X, \mathcal{A}, m, T)$ acting on the standard Hilbert bundle $\int^{\oplus} H_{x} d m(x)$ is a measurable map from $(X, \mathcal{A})$ to $\mathcal{U}(H)(H$ being the constant fiber space), $x \mapsto U_{x}$, and its action is defined as follows: for $v(x) \in \int^{\oplus} H_{x} d m(x)$, we set

$$
U v(x)=U_{T^{-1}(x)} v\left(T^{-1}(x)\right) .
$$

We then call $U$ a standard unitary cocycle.

On a general Hilbert bundle $\int^{\oplus} H_{x} d m(x)$, a unitary cocycle $U$ will be a measurable mapping from $(X, \mathcal{A}), x \mapsto U_{x}$, where for almost every $x$, $U_{x}$ is a unitary isomorphism from $H_{x}$ to $H_{T x}$. Given two unitary Hilbert bundles $\int^{\oplus} H_{1, x} d m(x)$ and $\int^{\oplus} H_{2, x} d m(x)$ and two unitary cocycles above $(X, \mathcal{A}, m, T), U_{1}$ and $U_{2}$, acting on $\int^{\oplus} H_{1, x} d m(x)$ and $\int^{\oplus} H_{2, x} d m(x)$ respectively, they are said to be cohomologous if there exists an isomorphism $W=W_{x}, x \in X$, from $\int^{\oplus} H_{1, x} d m(x)$ to $\int^{\oplus} H_{2, x} d m(x)$ such that

$$
U_{1, x}=W_{T(x)}^{-1} U_{2, x} W_{x} \text {. }
$$


(This is just expressing $U_{1}$ in the isomorphic Hilbert bundle given by the family $W_{x}$.)

Note that if $X$ is the one-point space endowed with the Dirac measure, a cocycle is just a single unitary operator, and cohomology reduces to unitary equivalence. The purpose of the relative spectral theory is to find, when possible, cohomology invariants for these cocycles. It does not seem realistic to hope, in general, for a complete set of invariants, as was the case in the "absolute" situation. However, in the relative spectral theory framework, the relatively discrete finite-dimensional spectrum is well understood (see [5], [14]) and has been used in the ergodic-theoretical proof by H. Furstenberg of the theorem of Szemerédi on the existence of arithmetic progressions in subsets of the integers with upper positive density. A number of questions occur in the general framework and we shall mention some of them. We are going to give two results (Propositions 1 and 2) which belong to the relative spectral theory and which have some relevance to the following two open problems:

(1) Consider two $K$-automorphisms $\left(X_{1}, \mathcal{A}_{1}, m_{1}, T_{1}\right)$ and $\left(X_{2}, \mathcal{A}_{2}, m_{2}, T_{2}\right)$ which have the same entropy $a$. We recall that a joining $\lambda$ of these two systems is a $T_{1} \times T_{2}$-invariant measure on $X_{1} \times X_{2}$ whose marginals are $m_{1}$ and $m_{2}$. The question, due to $\mathrm{B}$. Weiss, is whether there exists a joining $\lambda$ such that the system $\left(X_{1} \times X_{2}, T_{1} \times T_{2}, \lambda\right)$ is a $K$-automorphism and keeps $a$ as its entropy. In ordinary words, is it possible to find a $K$-joining of $T_{1}$ and $T_{2}$ which does not increase entropy?

(2) Consider a Bernoulli shift $(X, \mathcal{A}, m, T)$ and a factor algebra $\mathcal{B}$ (a $T$ invariant subalgebra of $\mathcal{A}$ ). (Here, and in the future, by abuse of language, subalgebras are really sub- $\sigma$-algebras.) We form the relatively independent joining of $T$ with itself above $\mathcal{B}$ (that is, we endow $X \times X$ with the $T \times T$ invariant measure which gives to sets of the form $A_{1} \times A_{2}$ the measure $\left.\int E^{\mathcal{B}} 1_{A_{1}} E^{\mathcal{B}} 1_{A_{2}} d m\right)$. Assuming that this relatively independent joining is ergodic, is it automatically $K$ ? If not, what kind of 0 -entropy transformation can appear as a factor of such a joining? If it is $K$, is it Bernoulli?

2. We recall a theorem of Rokhlin: Let $(X, \mathcal{A}, m, T)$ be an ergodic dynamical system and $\mathcal{B}$ a $T$-invariant subalgebra of $\mathcal{A}$. We denote by $\left(X_{\mathcal{B}}, \mathcal{B}, m, T\right)$ the system which is the restriction of the action of $T$ to $\mathcal{B}$. There is a Lebesgue measure space $(Y, \mathcal{C}, \mu)$, and a $\mathcal{B}$-measurable mapping from $X_{\mathcal{B}}$ to $\operatorname{Aut}(Y)$ (the group of measure preserving transformations of $(Y, \mathcal{C}, \mu)$ endowed with the Borel structure associated to the weak topology), $x \mapsto \psi_{x}$, such that the transformation $T_{\psi}$ on $X_{\mathcal{B}} \times Y$ equipped with the invariant measure $m \times \mu$ defined by

$$
T_{\psi}(x, y)=\left(T(x), \psi_{x}(y)\right)
$$


is isomorphic to $(X, \mathcal{A}, m, T)$ via an isomorphism which is the identity when restricted to $\mathcal{B}$. Furthermore, this representation is uniquely defined up to cohomology, that is, if $T_{\psi^{1}}$ is isomorphic to $T_{\psi^{2}}$ via an isomorphism which is the identity when restricted to $\mathcal{B}$, then there exists a $\mathcal{B}$-measurable mapping from $X_{\mathcal{B}}$ to $\operatorname{Aut}(Y), x \mapsto \phi_{x}$, such that

$$
\psi_{x}^{1}=\phi_{T x}^{-1} \psi_{x}^{2} \phi_{x} \quad \text { a.e. }
$$

We shall refer to $\psi_{x}$ as the Rokhlin cocycle associated to the factor $\mathcal{B}$ of $T$ (see, for instance, [6]). If one only considers the unitary action of this cocycle on $L^{2}(Y)$, one obtains a cocycle $U_{x}$ of unitary transformations $\left(U_{x}\right.$ is the unitary Koopman operator induced by $\psi_{x}$ ). We call $U_{x}$ the standard unitary Rokhlin cocycle associated to $\mathcal{B}$ or the unitary cocycle associated to $T_{\mathcal{B}}$, the restriction of $T$ to $\mathcal{B}$. The operator $U_{x}$ acts on $\int^{\oplus} H_{x} d m(x)$ (where $H_{x}$ is a copy of $L^{2}(Y)$ above every $\left.x \in X_{\mathcal{B}}\right)$.

Let us mention that there exist examples (due to A. Rosenthal, unpublished Ph.D. thesis) of two cohomologous Rokhlin cocycles above an irrational rotation which are constants (i.e. $\psi_{x}^{1}=T_{1}$ and $\psi_{x}^{2}=T_{2}, \psi^{1}$ and $\psi^{2}$ cohomologous) but $T_{1}$ and $T_{2}$ are non-isomorphic $K$-automorphisms with the same entropy. However, if one of the constants is isomorphic to a Bernoulli shift, then so must be the other. In the same way, due to results in the relative isomorphism theory, it has been proved by the author (unpublished) that if two unitary operators, viewed as cocycles above an ergodic transformation $T$, which are constant almost everywhere, are cohomologous, and if one of the constants has absolutely continuous spectral measure, then so does the other. Let us also mention that there are examples, due to Ornstein [10], where the Rokhlin cocycle associated to a factor $\mathcal{B}$ is not cohomologous to a constant transformation, but where the associated unitary cocycle is cohomologous to a constant.

Although it is really the same as the Rokhlin cocyle, we slightly change our viewpoint to introduce the notion of (ir)reducibility for a cocycle (of measure preserving transformations, or unitary) defined above an ergodic measure preserving transformation. It will play an important role in the future.

Definition. Let $(X, \mathcal{A}, m, T)$ be an ergodic dynamical system, $(Y, \mathcal{B}, \mu)$ a Lebesgue space and $\psi_{x}$ a cocycle of measure preserving transformations acting on $Y$. This gives rise to a transformation $T_{\psi}$ of $(X \times Y, \mathcal{A} \times \mathcal{B}, m \times \mu)$, $(x, y) \mapsto\left(T(x), \psi_{x}(y)\right)$. The cocycle $\psi_{x}$ is said to be reducible if there is a subalgebra $\mathcal{C} \subset \mathcal{B}$ and a cocycle $\phi_{x}$ such that $T_{\phi_{T x}^{-1} \psi_{x} \phi_{x}}$ leaves $\mathcal{A} \otimes \mathcal{C}$ invariant; it is said to be reducible to a constant if there exists a transformation $W$ of $Y_{\mathcal{C}}$ such that the restriction of $\phi_{T x}^{-1} \psi_{x} \phi_{x}$ to $\left(Y_{\mathcal{C}}, \mathcal{C}, \mu\right)$ is cohomologous to the constant $W$. In other words, $\psi_{x}$ is reducible if there exists a $T_{\psi}$-invariant 
algebra $\mathcal{A}_{1}$ such that $\mathcal{A} \subsetneq \mathcal{A}_{1} \subsetneq \mathcal{A} \times \mathcal{B}$; and it is reducible to a constant if, restricted to $\mathcal{A}_{1}, T_{\psi}$ is isomorphic to $T \times W$ via an isomorphism which is the identity when restricted to $\mathcal{A}$. A cocycle which is not reducible is said to be irreducible.

We now give the unitary version of this definition. Let $(X, \mathcal{A}, m, T)$ be an ergodic dynamical system, $U=U_{x}, x \in X$, a unitary cocycle acting on a Hilbert bundle $\int^{\oplus} H_{x} d m(x)$. We recall that this action is defined on the Hilbert bundle $\int^{\oplus} H_{x} d m(x)$ by $U(v)(x)=U_{T^{-1}(x)} v\left(T^{-1}(x)\right)$. The cocycle $U_{x}$ is said to be reducible if there exists a measurable field $H_{1, x}$, where for a.e. $x$, $H_{1, x}$ is a closed subspace of $H_{x}$, not equal to $H_{x}$ and not equal to 0 , such that $U_{x}\left(H_{1, x}\right)=H_{1, T(x)}\left(\int^{\oplus} H_{1, x} d m(x)\right.$ is then called an invariant subbundle $)$. If there is no such field, the cocycle $U$ is said to be irreducible. It is said to be reducible to a constant if the restriction of $U_{x}$ to $\int^{\oplus} H_{1, x} d m(x)$ is cohomologous to a constant unitary operator $W$. Two subbundles $\int^{\oplus} H_{i, x} d m(x)$, $i=1,2$, are said to be orthogonal if $H_{1, x} \perp H_{2, x}$ a.e.

We prove a first lemma which shows that there are very strong analogies between the relative spectral theory and the absolute one.

Lemma 0. Let $(X, \mathcal{A}, m, T)$ be ergodic, and $U=U_{x}, x \in X$, a unitary cocycle acting on a Hilbert bundle $\int^{\oplus} H_{x} d m(x)$. Given two invariant subbundles $\int^{\oplus} H_{1, x} d m(x)$ and $\int^{\oplus} H_{2, x} d m(x)$ which are not orthogonal, there exist two non-trivial invariant subbundles $\int^{\oplus} H_{i, x}^{\prime} d m(x)$ of $\int^{\oplus} H_{i, x} d m(x)$, $i=1,2$, respectively such that the restrictions of $U$ to $\int^{\oplus} H_{i, x}^{\prime} d m(x), i=1,2$, are cohomologous.

Proof. Given a section $v_{i}(x)$ in $\int^{\oplus} H_{i, x} d m(x)$, we denote by $H_{v_{i}}$ the $L^{2}$ closure of the $L^{\infty}(X)$-submodule generated by $U^{n} v_{i, x}, n \in \mathbb{Z}$. The hypothesis implies that there exist two sections $v_{i}(x)$ in $\int^{\oplus} H_{i, x} d m(x), i=1,2$, such that for almost every $x, v_{2}(x)$ is the projection of $v_{1}(x)$ on $H_{2, x}$; both sections are almost everywhere $\neq 0$, as a consequence of the ergodicity of $T$. We consider the projection operators $T_{x}$ from $H_{v_{1}, x}$ on $H_{v_{2}, x}$ $\left(H_{v_{i}}=\int^{\oplus} H_{v_{i}, x} d m(x), i=1,2\right)$. Clearly they satisfy $T_{T x} U_{x}=U_{x} T_{x}$ (in particular, $\left.T_{x}\left(v_{1}(x)\right)=v_{2}(x)\right)$. For every $x, T_{x}^{*}$ is the projection operator from $H_{v_{2}, x}$ to $H_{v_{1}, x}$. Let $H_{v_{2}, x}^{\prime}$ be the orthogonal complement in $H_{v_{2}, x}$ to the kernel of $T_{x}^{*}$. It is now true that $U_{x} T_{x}^{*}=T_{T x}^{*} U_{x}$ (these two operators send $H_{2, x}$ to $\left.H_{1, T x}\right)$ and, as a consequence, $U_{x} T_{x}^{*} T_{x}=T_{T x}^{*} T_{T x} U_{x}$ and, for every $k \in \mathbb{N}, U_{x}\left(T_{x}^{*} T_{x}\right)^{k}=\left(T_{T x}^{*} T_{T x}\right)^{k} U_{x}$ (easily checked). Therefore, denoting $\left|T_{x}\right|=\left(T_{x}^{*} T_{x}\right)^{1 / 2}$, we have $U_{x}\left|T_{x}\right|=\left|T_{T x}\right| U_{x}$. Let $H_{v_{1}}^{\prime}$ be the orthogonal complement in $H_{v_{1}}$ to the kernel of $T_{x}$. It is clear that $T_{x}^{*} T_{x}$, and hence $\left(T_{x}^{*} T_{x}\right)^{1 / 2}$, commutes with $U_{x}$. Thus, if $T_{x}=V_{x}\left|T_{x}\right|$ is the polar decomposition of $T_{x}$, then $U_{x} V_{x}\left|T_{x}\right|=V_{T x}\left|T_{T x}\right| U_{x}=V_{T x} U x\left|T_{x}\right|$ where $V_{x}$ is a field 
of unitary operators sending $H_{v_{1}, x}^{\prime}$ onto $H_{v_{2}, x}^{\prime}$. This gives the announced cohomology.

As an easy corollary, which should, in fact, have been part of [5], let us mention the following.

Corollary 1. Let an ergodic dynamical system $(X, \mathcal{A}, m, T)$ be given together with a non-trivial invariant subalgebra $\mathcal{B}$. Consider the standard unitary Rokhlin cocycle $U=U_{x}, x \in X_{\mathcal{B}}$, acting on $\int^{\oplus} H_{x} d m(x)\left(H_{x}\right.$ a copy of $L^{2}$ of the fiber space above every $\left.x\right)$. Assume that $\int^{\oplus} H_{i, x} d m(x)$, $i=1,2$, are two $U$-invariant finite-dimensional irreducible subbundles of $\int^{\oplus} H_{x} d m(x)$ and let $U_{1, x}$ and $U_{2, x}$ be the two finite-dimensional unitary cocycles corresponding to the restrictions of $U$ to these two subbundles (relative eigenvalues). Then either the two subbundles are orthogonal, or the cocycles $U_{i, x}, i=1,2$, are cohomologous.

This is immediate from Lemma 0 .

One specific feature of the relative situation is that there can exist irreducible subbundles of infinite dimension. Let us say that a unitary cocycle $U_{x}$ above $(X, \mathcal{A}, m, T)$ acting on $\int^{\oplus} H_{x} d m(x)$ has purely infinite discrete spectrum if there exists a family of $U$-invariant subbundles $\int^{\oplus} H_{i, x} d m(x), i \in \mathbb{N}$, pairwise orthogonal, such that (1) $\int^{\oplus} H_{x} d m(x)=\sum_{i=1}^{\infty} \int^{\oplus} H_{i, x} d m(x)$, (2) the restriction of the action of $U_{x}$ to $\int^{\oplus} H_{i, x} d m(x)$ is irreducible for all $i \in \mathbb{N}$, and (3) for all $i \in \mathbb{N}, \operatorname{dim} H_{i, x}=\infty$. (Note that we did not assume that the restrictions of the actions of $U_{x}$ to different invariant subbundles were necessarily inequivalent.)

If a transformation $(X, \mathcal{A}, m, T)$ has finite entropy and is $K$ and not Bernoulli, given a full entropy Bernoulli factor $\mathcal{B}$, the Rokhlin cocycle above $\mathcal{B}$ is irreducible. However, it is not known whether one can find a full entropy Bernoulli factor $\mathcal{B}$ for which the Rokhlin unitary cocycle is irreducible, or even has purely infinite discrete spectrum.

Another interesting issue is the following: Consider again a $K$-non-Bernoulli transformation with entropy $a$. Consider a full entropy Bernoulli factor and the associated unitary Rokhlin cocycle $U$. Is it possible to find, inside the Bernoulli shift of entropy $a$, a full entropy factor such that the associated unitary Rokhlin cocycle $V$ is cohomologous to $U$ ? (In other words, are there spectral characterisations of the property of being $K$ and not Bernoulli?) In this direction, let us mention the following theorem of Rudolph [11]: if the Rokhlin unitary cocycle above a full entropy Bernoulli factor of a transformation has a relative discrete finite-dimensional spectrum (that is, the associated Hilbert bundle is the direct sum of finite-dimensional invariant irreducible subbundles), then the transformation is Bernoulli. 
We now prove two results concerning the question of B. Weiss mentioned above. Let us first observe the following (we keep the notations used in formulating the question (1)). Let $\lambda$ be a joining of $\left(X_{1}, \mathcal{A}_{1}, m_{1}, T_{1}\right)$ and $\left(X_{2}, \mathcal{A}_{2}, m_{2}, T_{2}\right)$ constructed as the relatively independent joining over a full entropy Bernoulli factor $\mathcal{B}$ (obtained by choosing an isomorphism between two full entropy Bernoulli factors of $T_{1}$ and $T_{2}$ ). Because of the preceding theorem of Rudolph, $T_{1}$ and $T_{2}$ can be assumed to be both relatively weakly mixing with respect to this full entropy Bernoulli factor. Therefore, for $\lambda$, the $T_{1} \times T_{2}$ action is weakly mixing. If the joining constructed is not $K$, then the action of $T_{1} \times T_{2}$ restricted to the Pinsker algebra $\mathcal{C}$ is also weakly mixing. Due to the fact that 0 -entropy transformations are disjoint from $K$-automorphisms [4], $\mathcal{C}$ is independent of $\mathcal{A}_{1} \times X_{2}$ and $X_{1} \times \mathcal{A}_{2}$ respectively. If we consider the following three factor algebras of the action of $T_{1} \times T_{2}$ equipped with the joining measure $\lambda: \mathcal{A}_{1} \times X_{2}, X_{1} \times \mathcal{A}_{2}$ and $\mathcal{B} \times \mathcal{C}$, relative to $\mathcal{B}$, they have relative 0 entropy, are pairwise relatively independent, but are not globally relatively independent. Therefore we are faced with a relative version of an "absolute" problem which is still unsolved: Does there exist a joining of three weakly mixing 0-entropy transformations such that all its 2-marginals are independent, but it is not the product joining?

Proposition 0. Let $\left(X_{1}, \mathcal{A}_{1}, m_{1}, T_{1}\right)$ be a $K$-automorphism of entropy $\log 2$ and $(Y, \mathcal{B}, \mu, S)$ be the $T, T^{-1}$ transformation. The transformation $S$ is given, with $T$ denoting the Bernoulli 2-shift, by the following skew-product above $T$ : in the 2 -shift $T$ on the product space $((+1)(-1))^{\mathbb{Z}}$ with associated algebra $\mathcal{B}_{1}$, let $f(x)$ be the function which takes the values +1 or -1 according to whether $x_{0}=+1$ or $x_{0}=-1\left(x=\left(x_{i}\right), i \in \mathbb{Z}\right)$ and let $S$ be defined on the product $\mathcal{B}_{1} \otimes \mathcal{B}_{1}$ equipped with the product measure by $S(x, y)=\left(T x, T^{f(x)} y\right)$. In this situation there is an entropy preserving $K$ joining $\lambda$ of $T_{1}$ and $S$.

Proof. In fact, we only need to choose any full entropy Bernoulli factor of $T_{1}$ relative to which $T_{1}$ is weakly mixing. As $T_{1}$ and $S$ have the same entropy, this full entropy factor is isomorphic to $T$. We fix one isomorphism and we then take for $\lambda$ the relatively independent joining of $T_{1}$ and $S$ above $T$. Endowed with this joining measure $\lambda, T_{1} \times S$ will always be $K$. The proof is a direct consequence of the result of D. Rudolph ([12, Corollary 8]) which says that if the $K$ property does not hold, then, considering $S$ as an extension of $T_{1}$ (for the joining measure $\lambda$ which we have just defined), the function $f$ has to be cohomologous to a constant with a transfer function measurable with respect to $\mathcal{A}_{1}$. But due to the spreading of the distributions of the sums $\sum_{i=1}^{n} f\left(T^{i} x\right)$ the cohomology equation cannot be solved, even when the transfer function is allowed to be only $\mathcal{A}_{1}$-measurable. 
Proposition 1. Let $\left(X_{1}, \mathcal{A}_{1}, m_{1}, T_{1}\right)$ and $\left(X_{2}, \mathcal{A}_{2}, m_{2}, T_{2}\right)$ be two $K$ automorphisms with the same entropy. Let there be given two full entropy Bernoulli factors $\mathcal{B}_{1}$ and $\mathcal{B}_{2}$ of $T_{1}$ and $T_{2}$ respectively such that the unitary Rokhlin cocycles associated to the actions of $T_{1}$ over $\mathcal{B}_{1}$ and of $T_{2}$ over $\mathcal{B}_{2}$ have purely infinite discrete spectrum. The relatively independent joining of $T_{1}$ and $T_{2}$ above their common full entropy Bernoulli factor $\mathcal{B}$ is $K$.

Proof. We again denote by $\lambda$ the joining which has just been defined. Assume that on $X_{1} \times X_{2}$ equipped with $\lambda, T_{1} \times T_{2}$ has a 0 -entropy factor algebra $\mathcal{C}$. Let $H_{i, x}^{1}$ and $H_{i, x}^{2}, i \in \mathbb{N}$, be the infinite-dimensional invariant irreducible subbundles for the Rokhlin cocycles associated to the action of $T_{1}$ and $T_{2}$ respectively above $\mathcal{B}$. Let us consider the unitary Rokhlin cocycle corresponding to the action of $T_{1} \times T_{2}$ restricted to $\mathcal{A}_{1} \otimes \mathcal{C}$ above $\mathcal{B}$. The only discrete part in the decomposition of this unitary cocycle into irreducible components is $\sum_{i=1}^{\infty} H_{i, x}^{1} \otimes 1_{\mathcal{C}}$ (here we have used that if $K_{x}^{\mu_{1}}$ and $K_{x}^{\mu_{2}}$ are one-dimensional invariant subbundles associated to the Rokhlin cocycle corresponding to the action of $T_{1} \times T_{2}$ on $\mathcal{C}$ above $\mathcal{B}$ and on which this action is multiplication by $\mu_{1}$ and $\mu_{2}$ respectively, then $H_{1, x}^{1} \otimes K_{x}^{\mu_{1}}$ and $H_{1, x}^{1} \otimes K_{x}^{\mu_{2}}$ are both irreducible and inequivalent if $\mu_{1}$ and $\mu_{2}$ are distinct). We have also used the fact that the restriction of $T_{1} \times T_{2}$ to $\mathcal{C}$ is weakly mixing. But the bundle $\sum_{i=1}^{\infty} H_{i, x}^{1} \otimes 1_{\mathcal{C}}$ is orthogonal to $\sum_{i=1}^{\infty} H_{i, x}^{2}$. Since $L^{2}\left(\mathcal{A}_{1} \otimes \mathcal{C}\right)$ is not orthogonal to $L^{2}\left(\mathcal{A}_{2}\right)$, Lemma 0 shows that the unitary cocycle associated to the action of $T_{2}$ above $\mathcal{B}$ does not have a purely discrete spectrum. This contradiction implies that $\mathcal{C}$ is the trivial algebra.

It is very likely that more is true and that in fact the following can be shown to hold with not much difficulty: If $T_{1}$ and $T_{2}$ are two $K$-transformations with the same entropy such that there exists a full entropy Bernoulli factor of $T_{1}$ relative to which the unitary Rokhlin cocycle is not cohomologous to a constant having absolutely continuous spectrum, for any full entropy Bernoulli factor of $T_{2}$ relative to which $T_{2}$ is weakly mixing, the relatively independent joining defined as above will be $K$. In view of Host's theorem [7], this is as much as what can be hoped for, using (relative) spectral considerations.

3. We now study gaussian extensions associated to a unitary cocycle.

We first recall that, a separable $H$ being given, there exists a Lebesgue space $(Y, \mathcal{B}, \mu)$ such that $L^{2}(Y)$ can be identified to the sum

$$
1+\sum_{n \geq 1} H^{n \odot}
$$

of the symmetric tensor powers of $H$ in such a way that to every unitary operator $U$ acting on $H$, one can associate a measure preserving transfor- 
mation $\tau_{U}$ acting on $Y$ such that the unitary action of $\tau_{U}$ leaves every $H^{n \odot}$, $n \geq 1$, invariant, and restricted to $H^{n \odot}$ it is exactly $U^{n \odot}$ (in particular, the unitary action of $\tau_{U}$ on $H$ is exactly $U$; $\tau_{U}$ is the gaussian automorphism associated to $U$, see [1]). The entropy of $\tau_{U}$ is known: either the spectral measure of $U$ is singular and the entropy of $\tau_{U}$ is 0 , or it has an absolutely continuous component and the entropy of $\tau_{U}$ is $+\infty$ (see [2]).

If we are given $(X, \mathcal{A}, m, T)$ and a standard unitary cocycle $U=U_{x}$, $x \in X$, above $T$ acting on $\int^{\oplus} H_{x} d m(x)$ where $H_{x}$ is a copy of a fixed $H$ above every $x$, we can define $T_{\tau_{U}}$ acting on $(X \times Y, \mathcal{A} \otimes \mathcal{B}, m \times \mu)$ by $(x, y) \mapsto\left(T x, \tau_{U_{x}}(y)\right)$. We call $T_{\tau_{U}}$ the gaussian extension of $T$ by the standard unitary cocycle $U$. Restricted to a proper subspace of $L^{2}(Y)$, the unitary cocycle associated to this extension $T_{\tau_{U}}$ of $T$ acts exactly as $U$. We are going to show that for such gaussian skew-products, the relative entropy of the gaussian extension given the base is also 0 or $+\infty$. Furthermore, in case the entropy is positive, the cocycle $U$ is reducible to a constant which has an absolutely continuous part in its spectrum.

Now we prove the functoriality of the Rokhlin cocycle.

Lemma 1. Let $(X, \mathcal{A}, m, T)$ and a T-invariant subalgebra $\mathcal{B}$ of $\mathcal{A}$ be given. Let $\psi_{x}$ be the associated Rokhlin cocycle (acting on $(Y, \mathcal{C}, \mu)$ ). Let $\mathcal{D}$ be another $T$-invariant algebra which contains $\mathcal{B}$ as a subalgebra. Then there exists a cocycle $\phi_{x}$ above $T_{\mathcal{B}}$ and a subalgebra $\mathcal{E}$ of $\mathcal{C}$ such that $T_{\mathcal{B}, \phi_{T x}^{-1} \psi_{x} \phi_{x}}$ restricted to $\mathcal{B} \times \mathcal{E}$ is isomorphic to the restriction of $T$ to $\mathcal{D}$ via an isomorphism which is the identity when restricted to $\mathcal{B}$. We call $\phi_{\text {Tx }}^{-1} \psi_{x} \phi_{x}$ the $\mathcal{D}$-straightened version of $\psi$, and $\mathcal{D}_{Y}$ the associated fiber subalgebra.

Proof. This is an immediate application of the unicity of the Rokhlin cocycle, up to cohomology. We consider the Rokhlin cocycle $\psi_{1, x}$ associated to the restriction of $T$ to $\mathcal{D}$ above $\mathcal{B}$ (acting on $Y_{1}$ ) and the Rokhlin cocycle $\psi_{2, x, y_{1}}$ (acting on $Y_{2}$ ) for the action of $T$ on $\mathcal{A}$ above $X_{\mathcal{B}} \times Y_{1}$. A model for the Rokhlin cocycle associated to the action of $T$ on $\mathcal{A}$ above $\mathcal{B}$ is therefore $\psi_{1, x} \times \psi_{2, x, y_{1}}$, given that the fiber is $Y_{1} \times Y_{2}$. (The fiber transformations are themselves Rokhlin skew-products.) This last cocycle is cohomologous to $\psi_{x}$ by $\phi_{x}$, which provides the announced structure.

We recall the notion of relative Pinsker algebra. Let $(X, \mathcal{A}, m, T)$ be an ergodic transformation and $\mathcal{B}$ a $T$-invariant subalgebra of $\mathcal{A}$. The greatest subalgebra $\hat{\mathcal{B}}$ of $\mathcal{A}$ containing $\mathcal{B}$ and such that the entropy of $T$ acting on $\hat{\mathcal{B}}$ relative to $\mathcal{B}$ is 0 is the relative Pinsker algebra of the action of $T$ on $\mathcal{A}$ with respect to the algebra $\mathcal{B}$ (see [13]).

Proposition 2. Let $(X, \mathcal{A}, m, T)$, with $T$ ergodic, be endowed with an invariant subalgebra $\mathcal{B}$. Let $\psi_{x}$ and $U=U_{x}, x \in X$ be respectively the Rokhlin and unitary Rokhlin cocycles associated to $\mathcal{B}$. If the relative Pinsker 
algebra $\hat{\mathcal{B}}$ is not equal to $\mathcal{A}$, then the cocycle $U$ is reducible and, on the subspace on which it acts as a constant unitary operator, this operator has countable Lebesgue spectrum.

Proof. We first assume that the action of $T$ has finite relative entropy with respect to $\mathcal{B}$. There is therefore a finite relative generator $P$ for the action of $T$ on $\mathcal{A}$, and denoting by $\hat{\mathcal{B}}$ the relative Pinsker algebra, we have

$$
T^{-n} P^{-} \vee \hat{\mathcal{B}} \searrow \hat{\mathcal{B}} \quad \text { and } \quad T^{n} P^{-} \vee \hat{\mathcal{B}} \nearrow \mathcal{A} \quad \text { as } n \rightarrow+\infty .
$$

(see [13]; we recall that $P^{-}=\bigvee_{n=-1}^{-\infty} T^{n} P$ ). We prove that the unitary Rokhlin cocycle $U_{2}=U_{2, x}$ above $\hat{\mathcal{B}}$ is cohomologous to a constant unitary operator with countable Lebesgue spectrum. This is a consequence of the following. Let $Y_{2}$ be the space on which the Rokhlin cocycle associated to $\hat{\mathcal{B}}$ acts. Let $H_{2, x}$ be a copy of $L_{2}\left(Y_{2}\right)$ above every $x \in X_{\hat{\mathcal{B}}}$. There exists a family of sections in $\int^{\oplus} H_{2, x} d m(x), x \mapsto v_{i, k}(x)(i \in \mathbb{N}, k \in \mathbb{Z})$, such that:

(1) the $L^{2}$ closure of the $L^{\infty}\left(X_{\hat{\mathcal{B}}}\right)$-module generated by the $v_{i, k}(x), i \in \mathbb{N}$, $k \in \mathbb{Z}$, is the whole of $\int^{\oplus} H_{2, x} d m(x)$.

(2) If $(i, k) \neq(j, l)$ then for almost every $x \in X_{\hat{\mathcal{B}}}, v_{i, k}(x)$ is orthogonal (as an element of $H_{2, x}$ ) to $v_{j, l}(x)$.

(3) For all $(i, k) \in \mathbb{N} \times \mathbb{Z}, U_{2, x}\left(v_{i, k}(x)\right)=v_{i, k+1}(T(x))$.

To construct these sections $v_{i, k}(x)$, we first consider, above almost every $x$, the subspaces $H_{x}$ of $H_{2, x}$ which are, with $P$ as before being a partition of $X_{\hat{\mathcal{B}}} \times Y_{2}, L^{2}\left(\right.$ trace of $P^{-}$on $\left.x \times Y_{2}\right) \ominus L^{2}$ (trace of $T^{-1}\left(P^{-}\right)$on $\left.x \times Y_{2}\right)$. These spaces have infinite dimension, vary measurably and we can therefore find a measurable family $v_{i, 0}(x), i \in \mathbb{N}$, which is orthonormal and forms, for almost every $x$, a basis of $H_{x}$. The fields $v_{i, k}$ are now defined inductively by

$$
U_{2, T^{-1}(x)} v_{i, k-1}\left(T^{-1}(x)\right)=v_{i, k}(x) \quad \text { or } \quad v_{i, k}(x)=U_{2}^{k} v_{i, 0}(x) .
$$

This way, (3) is automatically satisfied. Because of the choice of the $v_{i, 0}$ and of this last definition for the general $v_{i, k}$, we get (2). For the same reason, $(1)$ is a consequence of $(*)$.

We use Lemma 1 with $\hat{\mathcal{B}}$ playing the role of $\mathcal{C}$ and we can therefore assume that there is a subalgebra $\mathcal{C}_{1}$ of $Y$ such that for almost every $x$, $\psi_{x}$ leaves $\mathcal{C}_{1}$ invariant, and the restriction of $T_{\psi_{x}}$ to $\mathcal{B} \times \mathcal{C}_{1}$ is exactly the action of $T$ on $\hat{\mathcal{B}}$. It now follows from what has been done that $U_{x}$ restricted to $L^{2}\left(\mathcal{C}_{1}\right)^{\perp}$ is reducible to a constant unitary operator. In case the relative entropy of $T$ given $\mathcal{B}$ is infinite, one only needs to take an increasing sequence of finite partitions $P_{n}$ which converge to $\mathcal{A}$. An increasing limit of cocycles which are all cohomologous to a constant operator with countable 
Lebesgue spectrum remains of the same form. This finishes the proof of Proposition 2.

As an easy corollary, we obtain:

Proposition 3. Let $(X, \mathcal{A}, m, T)$ be an ergodic dynamical system and $\mathcal{B}$ be an invariant subalgebra of $\mathcal{A}$. If the unitary Rokhlin cocycle associated to $\mathcal{B}$ has purely infinite discrete spectrum, then the relative entropy of the action of $T$ conditioned on $\mathcal{B}$ is 0 .

Proposition 4. Let $(X, \mathcal{A}, m, T)$ be an ergodic dynamical system, $U$ a standard unitary cocycle, and $T_{\tau_{U}}$ the associated gaussian extension. If the relative entropy of $T_{\tau_{U}}$ given $\mathcal{A}$ is positive, then $U$ is reducible to a constant which has an absolutely continuous spectral measure, and this relative entropy is therefore infinite.

Proof. Since the relative entropy of $T_{\tau_{U}}$ given $\mathcal{A}$ is positive, the Pinsker algebra of $T_{\tau_{U}}$ relative to $\mathcal{A}$ is a strict factor of the algebra on which $T_{\tau_{U}}$ acts $(\mathcal{A} \otimes \mathcal{B})$. Let $\hat{\mathcal{A}}$ be this relative Pinsker algebra. We consider $\tau_{U_{i}}$, the $\hat{\mathcal{A}}$ straightened version of $\tau_{U}$ with associated fiber algebra $\hat{\mathcal{A}}_{Y}$. It follows from Lemma 1 and Proposition 2 that the unitary cocycle acting on $L^{2}\left(\hat{\mathcal{A}}_{Y}\right)^{\perp}$ (we call this last subspace $W$ ) is cohomologous to a constant which is a unitary operator with countable Lebesgue spectrum. If $H_{x}$ is orthogonal to $W$, this says that $H_{x} \subset L^{2}\left(\hat{\mathcal{A}}_{Y}\right)$, which implies that the relative Pinsker algebra of $T_{\tau_{U}}$ with respect to $\mathcal{A}$ is exactly $\mathcal{A} \otimes \mathcal{B}$, which contradicts the hypothesis. Therefore $H_{x}$ is not orthogonal to $W$, and Lemma 0 implies the conclusion.

Acknowledgements. Part of this work was done during a most pleasant stay of the author at the Center for Dynamics and Geometry of Penn State University whose support is gratefully acknowledged.

\section{References}

[1] I. P. Cornfeld, S. V. Fomin and Ya. G. Sinaï, Ergodic Theory, Springer, New York, 1982.

[2] T. de la Rue, Entropie d'un système dynamique gaussien: cas d'une action de $Z^{d}$, C. R. Acad. Sci. Paris Sér. I Math. 317 (1993), 191-194.

[3] J. Dixmier, Les algèbres d'opérateurs dans l'espace hilbertien, Gauthier-Villars, Paris, 1969.

[4] H. Furstenberg, Disjointness in ergodic theory, minimal sets, and a problem in Diophantine approximation, Math. Systems Theory 1 (1967), 1-49.

[5] - Ergodic behavior of diagonal measures and a theorem of Szemerédi on arithmetic progressions, J. Anal. Math. 31 (1977), 204-256.

[6] E. Glasner, Ergodic Theory via Joinings, Math. Surveys Monogr. 101, Amer. Math. Soc., Providence, RI, 2003.

[7] B. Host, Mixing of all orders and pairwise independent joinings of systems with singular spectrum, Israel J. Math. 76 (1991), 289-298. 
[8] A. Katok and J.-P. Thouvenot, Spectral properties and combinatorial constructions in ergodic theory, in: Handbook of Dynamical Systems, Vol. 1B, Elsevier, Amsterdam, 2006, 649-743.

[9] M. Lemańczyk, J.-P. Thouvenot and B. Weiss, Relative discrete spectrum and joinings, Monatsh. Math. 137 (2002), 57-75.

[10] D. S. Ornstein, Factors of Bernoulli shifts, in: Conference on Ergodic Theory and Topological Dynamics (Kibbutz Lavi, 1974), Israel J. Math. 21 (1975), 145-153.

[11] D. Rudolph, Classifying the isometric extensions of a Bernoulli shift, J. Anal. Math. 34 (1978), 36-60.

[12] - $\mathbb{Z}^{n}$ and $\mathbb{R}^{n}$ cocycle extensions and complementary algebras, Ergodic Theory Dynam. Systems 6 (1986), 583-599.

[13] J.-P. Thouvenot, Une classe de systèmes pour lesquels la conjecture de Pinsker est vraie, Israel J. Math. 21 (1975), 208-214.

[14] R. Zimmer, Ergodic actions with generalized discrete spectrum, Illinois J. Math. 20 (1976), 555-588.

J.-P. Thouvenot

L. P. M. A.

Boîte courrier 188

4, Place Jussieu

75252 Paris Cedex 05, France

E-mail: jean-paul.thouvenot@upmc.fr

Received 16 April 2009;

in revised form 12 June 2009 